\title{
A New Near-Infrared Reflectance Spectroscopy Method for High-Throughput Analysis of Oleic Acid and Linolenic Acid Content of Single Seeds in Oilseed Rape (Brassica napus L.) ${ }^{\dagger}$
}

\author{
Oliver Niewitetzki,${ }^{\ddagger}$ Peter Tillmann,${ }^{\S}$ Heiko C. Becker,${ }^{\ddagger}$ And \\ Christian Möllers* $*$ \\ *Department of Crop Sciences, Georg-August-Universität Göttingen, Von-Siebold-Strasse 8, 37075 \\ Göttingen, Germany and ${ }^{\S}$ VDLUFA Qualitätssicherung NIRS GmbH, Am Versuchsfeld 13, 34128 \\ Kassel, Germany
}

\begin{abstract}
The development of oilseed rape cultivars with a high content of oleic acid (18:1) and a low content of linolenic acid (18:3) in the seed oil is an important breeding aim. Oil of this quality is increasingly being sought by the food and the oleochemical industry. Since the oil quality is determined by the genotype of the seed, a selection can be performed among single seeds of segregating populations. For this purpose a high-throughput Near-Infrared Reflectance Spectroscopy (NIRS) method using an automated sample presentation unit for single seeds of oilseed rape and a spectrometer equipped with a photodiode array detector was developed. Single-seed analyses have been accomplished with a throughput of up to 800 seeds per hour. Seeds from segregating populations of different origin were analyzed by NIRS and gas chromatography. Calibration equations were developed and validated applying the Modified Partial Least Square regression (MPLS) and LOCAL procedure. In three independent validations, standard errors of prediction corrected for bias between $2.7 \%$ and $3.7 \%$ for oleic acid and $1.2 \%$ and $1.8 \%$ for linolenic acid were determined using MPLS. Similar results were obtained applying the LOCAL procedure. The results show that the new highthroughput method can be applied to predict the oleic acid and linolenic acid content of single seeds of oilseed rape.
\end{abstract}

KEYWORDS: Fatty acids; quality; single-seed NIRS; near-infrared reflectance spectroscopy; HOLL; high oleic; low linolenic; photodiode array detector

\section{INTRODUCTION}

Most parts of the world grow "canola"- or "double-low"quality type oilseed rape with low seed oil contents of erucic acid and low seed glucosinolate contents. The oil is acknowledged for its health benefits because of its high contents of the essential fatty acids linoleic acid (18:2) and linolenic acid (18:3) (1-3). However, those high contents of polyunsaturated fatty acids represent a problem if the oil is used for frying and baking purposes. Under these conditions, the oil oxidizes quickly and develops an unacceptable flavor. Furthermore, canola oil is used to a large extent for the production of margarine. For this, the oil is partially hydrogenated which leads to the formation of undesired trans fatty acids (3). In view of these shortcomings, mutation experiments were performed a long time ago to modify the fatty acid profile of oilseed rape (3). As a result of these experiments, cultivars with reduced contents of linolenic acid (LL, low linolenic) have been released starting from $1988(4,5)$. Additional mutation experiments led to the development of mutant lines with reduced linoleic acid and enhanced oleic acid content (18:1; HO,

\footnotetext{
${ }^{\dagger}$ In memorandum of Oliver Niewitetzki who passed away unexpectedly during the course of this research work.

*Corresponding author. E-mail: cmoelle2@gwdg.de.
}

high oleic). Conventional crossing and selection of this material has led to the development of HOLL quality type oilseed rape (3). HOLL quality type oilseed rape contains more than $70 \%$ oleic acid and less than 3\% linolenic acid in the seed oil. First annual spring cultivars and then also winter oilseed rape cultivars of HOLL quality have been released and are cultivated on a contract basis as an identity preserved (IP) crop in Canada and in Europe. In 2007, HOLL quality spring oilseed rape was grown in Canada on more than 700.000 ha (6). In Europe, the first HOLL quality winter oilseed rape cultivar Splendor was released in 2004 in France. In the meantime, additional cultivars with improved oil quality and yield were released (6). In 2007 and 2008, HOLL quality winter oilseed rape was cultivated on about 22.000 ha in Europe. In anticipation of a quickly rising demand for HOLL quality oil, with "Vistive", a separate marketing concept was developed (6).

Breeding HOLL quality oilseed rape is not an easy task because, in addition to the standard breeding aims, the new quality trait needs to be considered. Thereby, several mutant alleles need to be combined to achieve the desired HOLL quality. The high oleic acid trait is reported to be inherited by one major and a few additional minor genes $(7,8)$. The low linolenic acid trait is inherited by three genes (9). The large number of genes 
involved in the expression of the HOLL quality trait leads to complex segregation patterns in offspring generations. HOLL quality can be determined by near-infrared reflectance spectroscopy (NIRS), which, however, in routine applications requires about $2-3 \mathrm{~g}$ of seeds $(10)$. Since the HOLL quality is determined by the genotype of the embryo, F2 seeds of segregating populations can be analyzed by the half-seed method for their fatty acid composition (11). However, this is labor intensive and requires costly gas chromatographic analyses. Therefore, standard NIRS was downsized by using special adapters which allow scanning of individual seeds. Single-seed NIRS calibrations have been developed successfully for determining fatty acids, oil, protein, and total glucosinolate content $(12-15)$. In all those works, the Modified Partial Least Square regression method (MPLS) has been used for linear calibration equation development. If more than about 2000 spectra and pertinent reference values are available, calibration equations can also be developed with the nonlinear LOCAL approach $(16,17)$.

Although single-seed NIRS proved to work for HOLL quality type selection in segregating F2 seed populations (13), this system is still slow because of the required manual loading and unloading of the ring cups with single seeds and the sequential recording of the reflected wavelengths produced by a monochromator. In contrast to this, the more recent development of suitable photodiode array detectors allows simultaneous measurement of the light reflected from the samples. The development of highthroughput single-seed NIRS procedures for selection of quality traits has previously been reported for wheat and maize $(18,19)$.

The aim of the present study was to test a new automated sample presentation device (PPM unum) connected via optic fibers with a new NIRS spectrometer (ZEISS MCS 611) equipped with a photodiode array detector for the NIRS scanning of individual seeds of oilseed rape and to develop calibrations for oleic acid, linoleic acid, and linolenic acid. The intention was also to compare NIRS predictions applying the MPLS and LOCAL procedure.

\section{MATERIAL AND METHODS}

Seed Material Used for Calibration Development and Cross Validation. Genetic divergent winter oilseed rape seed populations of canola/' $00^{\prime}$-quality with varying contents of oleic acid and linolenic acid derived from different environments (locations and years) were obtained from the Department of Crop Sciences, Plant Breeding, Georg-AugustUniversität of Göttingen, as well as from breeding companies. Seeds used in the analysis were derived from the years 2001 to 2008. In addition to the Departments own material, five different German winter oilseed rape breeding companies provided seeds from 281 different segregating F2 seed populations. On the basis of their origin and their fatty acid composition, in total 3152 seeds were selected and used for calibration development (Table 1).

Seed Material Used for Independent Validation. From the 3152 seeds, three sets of seed spectra $(\mathrm{V} 1-\mathrm{V} 3)$ of different origins were one after the other removed from the calibration file and used for independent validation. The three sets consisted of the complete sets of seeds provided by two breeding companies (V2 with $n=295$ and V3 with $n=113$ ) and by the complete set of seeds harvested in 2008 at the two different locations Hohenlieth and Göttingen (V1 with $n=234)$. Seeds from the validation set V1 were obtained from plants preselected for high and low contents of oleic acid and linolenic acid in the previous year. Each time, the remaining spectra were used to develop new calibrations ( $\mathrm{Cl}$ to $\mathrm{C} 3$ ). The calibrations $\mathrm{C} 1$ to $\mathrm{C} 3$ were used to predict the fatty acid contents of the corresponding validation sets V1 to V3 with seed samples not represented in the calibration.

Automated Sample Presentation Unit (PPM unum) and Spectrometer ZEISS MCS 61. The PPM unum was constructed by the Ingenieurbüro Steps (Jena, Germany) and the VDLUFA Qualitäts-
Table 1. NIRS Calibration and Cross Validation Statistics for the Percentage of Oleic (18:1), Linoleic (18:2), and Linolenic Acid (18:3) in the Oil of Intact Single Seeds of Oilseed Rape ${ }^{a}$

\begin{tabular}{|c|c|c|c|c|c|c|c|c|}
\hline \multirow[b]{2}{*}{ fatty acid } & \multirow[b]{2}{*}{$N$} & \multicolumn{3}{|c|}{ gas chromatography (GC; \%) } & \multicolumn{2}{|c|}{ calibration } & \multicolumn{2}{|c|}{ cross validation } \\
\hline & & mean & SD & range & $R_{\mathrm{C}}^{2}$ & SEC (\%) & $R_{\mathrm{CV}}^{2}$ & SECV (\%) \\
\hline $18: 1$ & 2892 & 74.3 & 7.1 & $46.4-88.9$ & 0.88 & 2.4 & 0.87 & 2.6 \\
\hline $18: 2$ & 2898 & 12.8 & 5.5 & $2.4-31.7$ & 0.79 & 2.5 & 0.77 & 2.6 \\
\hline $18: 3$ & 2938 & 5.0 & 2.2 & $1.1-14.4$ & 0.69 & 1.3 & 0.67 & 1.3 \\
\hline
\end{tabular}

${ }^{a}$ Values are expressed as percentages of total fatty acids. $N$ is the number of seed samples considered in calibration development. Mean, standard deviation (SD), and range are GC data. SEC is the standard error of calibration, and SECV is the standard error of cross validation. $R_{C}^{2}$ and $R_{\mathrm{CV}}^{2}$ are the coefficient of determination in calibration and cross validation, respectively.

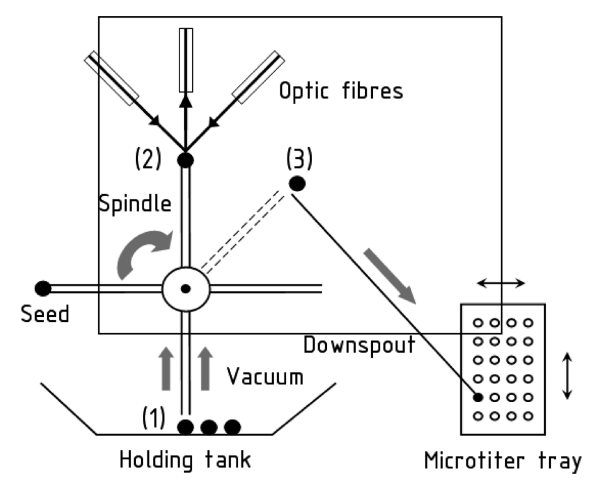

Figure 1. Principle of the automated sample presentation unit (PPM unum) for single seeds of oilseed rape, constructed by Ingenieurbüro Steps (Jena) and VDLUFA (Kassel).



Figure 2. Exterior view of the automated sample presentation unit PPM unum.

sicherung NIRS GmbH (Kassel, Germany). The principal functioning of the sample presentation of the PPM unum is depicted in Figure 1, and an exterior view of the equipment is shown in Figure 2. The tubes of a rotating spindle move through a holding tank containing seeds of oilseed rape. As the tubes are connected to a vacuum pump, single seeds are absorbed by the tubes (1) and are transported to a set of three optic fibers (2). The outer two fibers transmit white light of a halogen lamp to the sample seed, while the inner fiber collects the reflected light and transmits it to the spectrometer. The spectrometer ZEISS MCS 611 is equipped with a photodiode array detector that is able to record the full spectrum $(1340-2000 \mathrm{~nm})$ at once within milliseconds. Finally, the single seed is stripped off into a downspout (3) and arrives at a free position of a polystyrene $96 \mathrm{U}$ well microplate (greiner bio-one, Frickenhausen, Germany), which then moves automatically to the next free position. Precondition for an error-free and reliable measurement of the seeds is the availability of round seeds with a diameter of $1.8-2.3 \mathrm{~mm}$ (thousand seed weight $4.0-6.0 \mathrm{~g}$ ). Before measurement, seed samples were therefore graded according to their size by sieving, and seeds were put onto a ramp to separate angular from round shaped seeds. To allow an accurate assignment of the seed spectra stored in a file to their corresponding seeds deposited in 96-well microplates, seeds were mixed before measurement 
Table 2. NIRS Independent Validation Statistics for the Percentage of Oleic (18:1), Linoleic (18:2), and Linolenic Acid (18:3) in the Oil of Intact Single Seeds of Oilseed Rape ${ }^{a}$

\begin{tabular}{|c|c|c|c|c|c|c|c|c|c|c|}
\hline \multirow[b]{2}{*}{ fatty acid } & \multicolumn{3}{|c|}{ calibration } & \multicolumn{5}{|c|}{ independent validation gas chromatography (GC; \%) } & \multicolumn{2}{|c|}{ validation } \\
\hline & name & SECV & $R_{\mathrm{CV}}^{2}$ & name & $N$ & mean & SD & range & $\operatorname{SEP}(C)$ & $R_{\mathrm{V}}^{2}$ \\
\hline \multirow[t]{3}{*}{$18: 1$} & $\mathrm{C} 1$ & 2.6 & 0.85 & V1 & 234 & 72.0 & 12.1 & $49.3-86.1$ & 3.7 & 0.91 \\
\hline & $\mathrm{C} 2$ & 2.5 & 0.88 & V2 & 295 & 76.0 & 5.0 & $63.4-88.9$ & 2.7 & 0.74 \\
\hline & C3 & 2.5 & 0.86 & V3 & 113 & 60.9 & 7.6 & $46.3-71.9$ & 3.4 & 0.80 \\
\hline \multirow[t]{3}{*}{ 18:2 } & C1 & 2.5 & 0.77 & V1 & 234 & 13.7 & 8.3 & $2.7-29.0$ & 4.2 & 0.78 \\
\hline & $\mathrm{C} 2$ & 2.7 & 0.78 & V2 & 295 & 13.1 & 4.4 & $3.0-23.8$ & 2.5 & 0.67 \\
\hline & $\mathrm{C} 3$ & 2.6 & 0.75 & V3 & 113 & 21.2 & 6.2 & $11.1-31.7$ & 3.2 & 0.73 \\
\hline \multirow[t]{3}{*}{$18: 3$} & $\mathrm{C} 1$ & 1.3 & 0.62 & V1 & 234 & 6.0 & 3.8 & $1.6-12.5$ & 1.8 & 0.90 \\
\hline & $\mathrm{C} 2$ & 1.9 & 0.69 & V2 & 295 & 4.7 & 1.2 & $2.3-8.4$ & 1.2 & 0.33 \\
\hline & C3 & 1.3 & 0.65 & V3 & 113 & 7.7 & 2.6 & $3.2-17.0$ & 1.5 & 0.72 \\
\hline
\end{tabular}

${ }^{a}$ Values are given as a percentage of total fatty acids. Nis the number of seed samples in validation sets. Mean, standard deviation (SD), and range are GC data. SEP(C) is the standard error of prediction corrected for bias. $R_{\mathrm{CV}}^{2}$ and $R_{V}^{2}$ are the coefficient of determination and the coefficient of determination in cross validation, respectively.

with small plastic beads made of polyoxymethylene (POM; yellow color; diameter, $2 \mathrm{~mm}$; thousand kernel weight, $6 \mathrm{~g}$; specific gravity, $1.40 \mathrm{~g} / \mathrm{cm}^{3}$; Kugelfertigung Hoch KG, Hassfurt, Germany). The beads were measured together with the normal seeds and were deposited randomly on the microplates. Checking correct assignment of predicted results to spectra and seeds in the microplates was easy because of the yellow color of the beads, their characteristic NIRS spectrum, and their predicted nonsense values for oleic acid content.

Spectra Recording, Reference Analysis, and Calibration Development. Seeds were scanned individually by NIRS (1340-2000 nm), and reflectance spectra were recorded using the CORA software 3.2.1 (Fa. Carl Zeiss Jena $\mathrm{GmbH}$ ). Scanning time was set to $1 \mathrm{~s}$ per seed kernel, resulting in up to 20 seeds scanned per minute. In this second, five single scans were recorded by the software, which in turn were the average of three scans in the spectrometer each. Spectra files of the .spc-format were converted to the .NIR-format using a self-developed SPC2NIR converter. Subsequently, individual seeds were analyzed by gas chromatography (GC) for their fatty acid content according to ref 9 . Fatty acids are expressed as percent of the sum of all fatty acids. The following fatty acids were determined: palmitic acid (16:0), palmitoleic acid (16:1), hexadecadienoic acid (16:2), stearic acid (18:0), oleic acid (18:1), linoleic acid (18:2), linolenic acid (18:3), arachidic acid (20:0), gadoleic acid (20:1), behenic acid (22:0), and erucic acid (22:1). A standard rapeseed oil was used repeatedly during the course of the study to determine the standard gas chromatographic error. For oleic acid and linolenic acid, the error was $0.08 \%$ and $0.03 \%$, respectively. To determine the NIRS prediction error, 10 seeds of different size were each measured 10 times. The predicted results were then used to calculate the mean standard error, which was $0.41 \%$ for oleic acid and $0.19 \%$ for linolenic acid content. The calibration file was used to develop calibration equations with spectral absorbance information using WinISI II Project Manager 1.50 (Infrasoft International LLC, 1362 South Atherton St., State College, PA 16801, USA).

GLOBAL calibrations were developed using Modified Partial Least Squares regression analysis (MPLS) and cross validation techniques. Spectra were first treated with the scatter correction "SNV and Detrend". Then, the first derivative was used in combination with a gap of $4 \mathrm{~nm}$. The first and second smooth were set to 4 and $1 \mathrm{~nm}$, respectively. The maximum number of terms allowed by the software were 16, but the number of terms used ranged between 8 (linolenic acid) and 9 (oleic acid and linoleic acid). For cross validation, the suggested setting of four groups was used. The results of the calibration were checked by observing $t$ and global $\mathrm{H}$ $(\mathrm{GH})$ outliers. Outliers with $t>2.5$ and $\mathrm{GH}>10$ were not considered for calibration development. The number of outlier elimination passes was 2 . Calibration performance was assessed by standard error of calibration (SEC), coefficient of determination $\left(R_{\mathrm{C}}^{2}\right)$, standard error of cross validation (SECV), and coefficient of determination in cross validation $\left(R_{\mathrm{CV}}^{2}\right)$. The standard deviations (SD) and the means for the calibration sets as well as range of the calibration were taken from the WinISI software. Calibration performance in validation was assessed by standard error of performance corrected for bias $(\operatorname{SEP}(C))$ and by the coefficient of determination in validation $\left(R_{\mathrm{V}}^{2}\right)$.

Nonlinear LOCAL calibrations were developed using the routine provided by the WinISI software. A preliminary test was performed to determine the best setting for the LOCAL database. The test consisted of calculating the prediction error of LOCAL by changing the number of samples selected $(50,100,200$, and 300), the maximum number of factors used in PLS regression $(10,20)$, and the number of PLS factors excluded $(2,4)$. The following settings gave the best results: 200, 10, 2 . One outlier elimination pass was performed to exclude extreme spectral outliers with $t>3.0$ and $\mathrm{GH}>3$.

\section{RESULTS}

Provided that seed samples had a uniform round shape and a size of $1.8-2.3 \mathrm{~mm}$ in diameter, the automated sample presentation unit PPM Unum allowed the accurate scanning of 800 seeds per hour. Inadequate seed quality resulted in incorrect deposition of seeds in the microplate cavities (no seed or two seeds per cavity) which resulted in a throughput reduction. Unequivocal identification of the plastic beads mixed among the seed samples before measurement allowed quick checking of accurate assignment of spectra to seed.

In the complete calibration seed sample set, the oleic acid content ranged from $46 \%$ to $89 \%$ with a standard deviation of $7.1 \%$ (Table 1). Applying the GLOBAL MPLS calibration routine, the standard error of cross validation (SECV) was $2.6 \%$, and the coefficient of determination in cross validation $\left(R_{\mathrm{CV}}^{2}\right)$ was 0.87 . The ratio $\mathrm{SD} / \mathrm{SECV}$ was 2.7 . Linoleic acid content ranged from $2.4 \%$ to $32 \%$ with a standard deviation of $5.5 \%$. The SECV was $2.6 \%$, and the coefficient of determination in cross validation $\left(R_{\mathrm{CV}}^{2}\right)$ was 0.77 . Linolenic acid content ranged from $1.1 \%$ to $14 \%$ with a standard deviation of $2.2 \%$. The SECV was $1.3 \%$, and the coefficient of determination in cross validation $\left(R_{\mathrm{CV}}^{2}\right)$ was 0.67 . The ratio $\mathrm{SD} / \mathrm{SECV}$ was 2.1 for linoleic acid and 1.7 for linolenic acid.

In the independent validation, the three new calibrations $\mathrm{C} 1$ to C3 were not much different from the calibration based on the complete seed sample set (compare SECV and $R_{\mathrm{CV}}^{2}$ of Table 1 and Table 2). The three independent validation sets showed large differences in standard deviations and ranges for the individual fatty acids (Table 2). For oleic acid, the standard errors of prediction corrected for bias $(\mathrm{SEP}(\mathrm{C}))$ ranged between 2.7 and $3.7 \%$. The coefficients of determination in validation $\left(R_{\mathrm{V}}^{2}\right)$ varied from 0.74 to 0.91 (Table 2 and Figure 3). The SD/SEP(C) ratio ranged from 1.9 for validation set 2 to 3.3 for validation set 1 . In the first and in the third validation set V1 and V3, the standard error of prediction corrected for bias $(\operatorname{SEP}(C))$ was higher than the standard error in the calibration set (SEC; Table 1). For linoleic acid, the $\mathrm{SEP}(\mathrm{C})$ varied from 2.5 to $4.2 \%$, and the coefficients of determination in validation $\left(R_{\mathrm{V}}^{2}\right)$ ranged from 0.67 to 0.78 . The $\mathrm{SD} / \mathrm{SEP}(\mathrm{C})$ ratio ranged from 1.8 for validation set 2 to 2.0 for validation set 1 . Validation sets 1 and 3 gave 

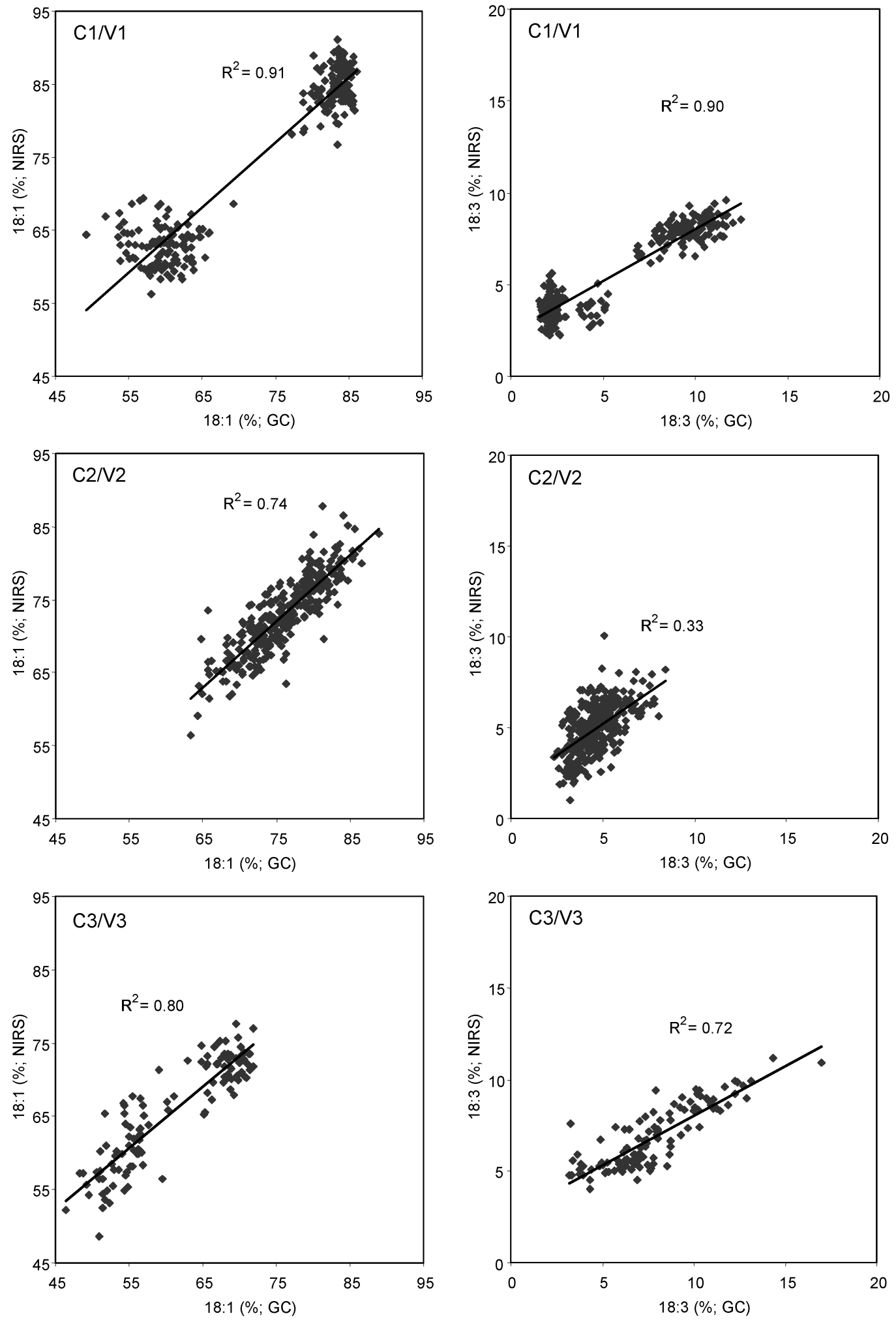

Figure 3. Scatterplot of the oleic acid (18:1) and linolenic acid (18:3) contents of individual seeds of oilseed rape as determined by GC and as predicted by single-seed NIRS. Depicted are the results of the independent validations of the three seed populations V1-V3 (see Table 2).

satisfying results for linolenic acid, but for $\mathrm{V} 2$, the $\mathrm{SEP}(\mathrm{C})$ was as large as the standard deviation, and the coefficient of determination in validation $\left(R_{\mathrm{V}}^{2}\right)$ was as low as 0.33 (Figure 3).

To compare the linear MPLS calibration with a nonlinear calibration approach, a LOCAL calibration was developed using the same data set. Following the elimination of outliers, between 3088 and 3095 seed samples remained in the database. The standard error of prediction $(\operatorname{SEP}(\mathrm{C}))$ ranged between $1.2 \%$ for linolenic acid and 3.0\% for oleic acid (Table 3 ). The coefficients of determination in validation $\left(R_{\mathrm{V}}^{2}\right.$, Table 3 ) were comparable to the results obtained in cross validation $\left(R_{\mathrm{CV}}^{2}\right.$, Table 1) using the GLOBAL-MPLS calibration approach. Also, coefficients of determination in validation $\left(R_{\mathrm{V}}^{2}\right)$ obtained in independent validations (Table 4) were similar to those obtained with the linear MPLS calibration (see Table 2). The percentages of samples for which the oleic acid and linolenic acid content have been 
Table 3. Results of the LOCAL Calibration for the Percentage of Oleic (18:1), Linoleic (18:2), and Linolenic Acid (18:3) in the Oil of Intact Single Seeds of Oilseed Rape ${ }^{a}$

\begin{tabular}{cccc}
\hline fatty acid & $N$ & $\operatorname{SEP}(\mathrm{C})$ & $R_{\mathrm{V}}^{2}$ \\
\hline $18: 1$ & 3091 & 3.0 & 0.84 \\
$18: 2$ & 3095 & 2.8 & 0.77 \\
$18: 3$ & 3088 & 1.2 & 0.71 \\
\hline
\end{tabular}

${ }^{a} N$ is the number of seed samples considered in the database. SEP $(C)$ is the standard error of prediction corrected for bias, and $R_{V}^{2}$ is the coefficient of determination in validation.

Table 4. Results of the LOCAL Calibration Approach in Independent Validation Using Three Different Seed Sample Sets for the Prediction of Oleic (18:1), Linoleic (18:2), and Linolenic Acid (18:3) in the Oil of Intact Single Seeds of Oilseed Rape ${ }^{a}$

\begin{tabular}{|c|c|c|c|c|c|c|c|}
\hline \multirow[b]{2}{*}{ fatty acid } & \multicolumn{3}{|c|}{ calibration } & \multicolumn{4}{|c|}{ validation } \\
\hline & name & $\operatorname{SEP}(C)$ & $R_{\mathrm{V}}^{2}$ & name & $N$ & $\operatorname{SEP}(C)$ & $R_{V}^{2}$ \\
\hline \multirow[t]{3}{*}{$18: 1$} & C1 & 3.0 & 0.81 & V1 & 234 & 3.3 & 0.93 \\
\hline & $\mathrm{C} 2$ & 3.2 & 0.83 & V2 & 295 & 2.5 & 0.76 \\
\hline & $\mathrm{C} 3$ & 3.1 & 0.81 & V3 & 113 & 3.3 & 0.83 \\
\hline \multirow[t]{3}{*}{$18: 2$} & $\mathrm{C} 1$ & 2.8 & 0.75 & V1 & 234 & 3.8 & 0.80 \\
\hline & $\mathrm{C} 2$ & 2.9 & 0.76 & V2 & 295 & 2.4 & 0.70 \\
\hline & $\mathrm{C} 3$ & 2.8 & 0.74 & V3 & 113 & 3.3 & 0.74 \\
\hline \multirow[t]{3}{*}{$18: 3$} & C1 & 1.2 & 0.66 & V1 & 234 & 1.9 & 0.84 \\
\hline & C2 & 1.3 & 0.71 & V2 & 295 & 1.0 & 0.35 \\
\hline & C3 & 1.2 & 0.69 & V3 & 113 & 1.5 & 0.67 \\
\hline
\end{tabular}

${ }^{a}$ Values are given as percentages of total fatty acids. Means, standard deviations (SD), and ranges are the same as in Table 2. Nis the number of seed samples in the validation sets. SEP $(C)$ is the standard error of prediction corrected for bias, and $R_{V}^{2}$ is the coefficient of determination in validation.

predicted correctly and with given deviations are shown in Figure 4. For oleic acid, 25\% of the samples were predicted correctly; $43 \%$ of the predicted samples had deviations of less than $1 \%$ oleic acid; and about $75 \%$ of the predicted samples had deviations of less than $2 \%$ oleic acid compared to the reference values. For linolenic acid, $26 \%$ of the samples were predicted correctly, and about $75 \%$ of the predicted samples had deviations of less than $1 \%$ linolenic acid compared to the reference values.

\section{DISCUSSION}

The fatty acid composition of an individual seed genotype not only is determined by its genotype but also is influenced by prevalent light and temperature conditions during its development and possibly by maternal factors $(20,21)$. The influence of those factors on the seed oil quality may vary with the position of the seed at the main, secondary, or even tertiary shoot. Furthermore, fatty acid composition may differ from plant to plant due to environmental conditions. Hence, F2 seed populations used for single-seed selection for oil quality should be derived from the main shoot only and should be analyzed separately for each F1 plant. Despite these shortcomings, selection for oil quality in HOLL oilseed rape breeding programs is done routinely by gas chromatographic analysis of the fatty acid composition of a single cotyledon detached from a germinated seedling (11). However, this method is laborious and costly. The automated single-seed NIRS method described in the present work has the potential to scan up to 800 seeds per hour. However, this throughput is only achieved with seed lots which have been graded according to their size $(1.8-2.3 \mathrm{~mm})$ and which are of round shape. Not welldeveloped and angular formed seeds led to faulty measurements. Well-developed round seeds are usually obtained from plants grown in the field, whereas greenhouse grown plants frequently yield small and angular shaped seeds. Therefore, for single-seed NIRS analysis, F2 seed populations derived from field grown plants should be used preferentially.

The standard method for the linear calibration equation development is the Modified Partial Least Square regression method (MPLS) $(10,13,15)$. Applying this method, the standard error of prediction corrected for bias $(\mathrm{SEP}(\mathrm{C}))$ obtained in independent validation comprises the errors caused by the gas chromatographic reference analysis and by the NIRS measurement. For oleic acid, this error ranged between 2.7 and $3.7 \%$ (Table 2). This compares well to previously obtained results using a manual loaded single-seed adapter in combination with a monochromator (SEP $=2.73 \%)(13)$ and appears acceptable considering the absolute variation of $26-37 \%$ in the three different validation sets. The $\mathrm{SD} / \mathrm{SEP}(\mathrm{C})$ ratio ranged from 1.9 for validation set 2 to 3.3 for validation set 1, indicating that an efficient selection is only possible, when there is a large variation for the trait under selection (22). For linolenic acid, the standard error of prediction corrected for bias ranged between 1.2 and $1.8 \%$, which is similar to the results obtained by Velasco et al. (13) $(\mathrm{SEP}=1.53 \%)$, but which appears quite high compared to the standard deviation of $1.2-3.8 \%$. However, the large range for linolenic acid indicates that nevertheless the selection of extreme phenotypes will be possible (see Figure 3). The largest variance for the traits high oleic and low linolenic can be expected in F2 seed populations derived from crosses between HOLL genotypes and current high-yielding Canola/00-quality type oilseed rape cultivars. Hence, such F2 seed populations should be used preferably for the single-seed NIRS selection. Furthermore, one should mind to analyze a sufficiently large number of seeds to make sure that there are homozygous recombinants. Those should show the largest variation in fatty acid composition and should be more easy to detect at a given prediction error. In the case of five loci being involved in the inheritance of the trait HOLL and no linkage, then already more than 3000 seeds need to be analyzed, to have a $95 \%$ probability for the presence of a seed which is homozygous for all five loci.

Since more than 3000 seed samples spectra and their pertinent reference values were included in the database, this allowed testing of the LOCAL procedure, a nonlinear approach for calibration development (refs 16,17, and references therein). With the available data set, the GLOBAL MPLS and the LOCAL procedure led to similar results (compare Tables 2 and 4). However, the prediction results of the LOCAL procedure may improve if new samples are included in the database because always only the spectral most similar subset of seed samples of the database ( $n=200$ in the present work) is used for the prediction of a new seed sample spectra. In contrast, the prediction results of the GLOBAL MPLS procedure are not likely to change much when new samples are included.

Regarding the application of the fast single-seed NIRS method in breeding HOLL-quality winter oilseed rape, there are in principle two approaches possible. First, between harvest in July and sowing in August, as many as possible segregating F2 seed populations are analyzed, and selected HOLL seed fractions are sown in the field. At onset of flowering, F2 plants are bagged to secure self-pollination, and harvested F3 seeds are analyzed for their seed oil quality applying the standard NIRS method. Second, the fast single-seed NIRS method is applied to perform a preselection of the desired HOLL types, and gas chromatographic fatty acid analyses of detached cotyledons are performed on a drastically reduced F2 seed number only. In this case, selected F2 plants are grown in the greenhouse, but in the right season they may also be transplanted later to the field. In either 

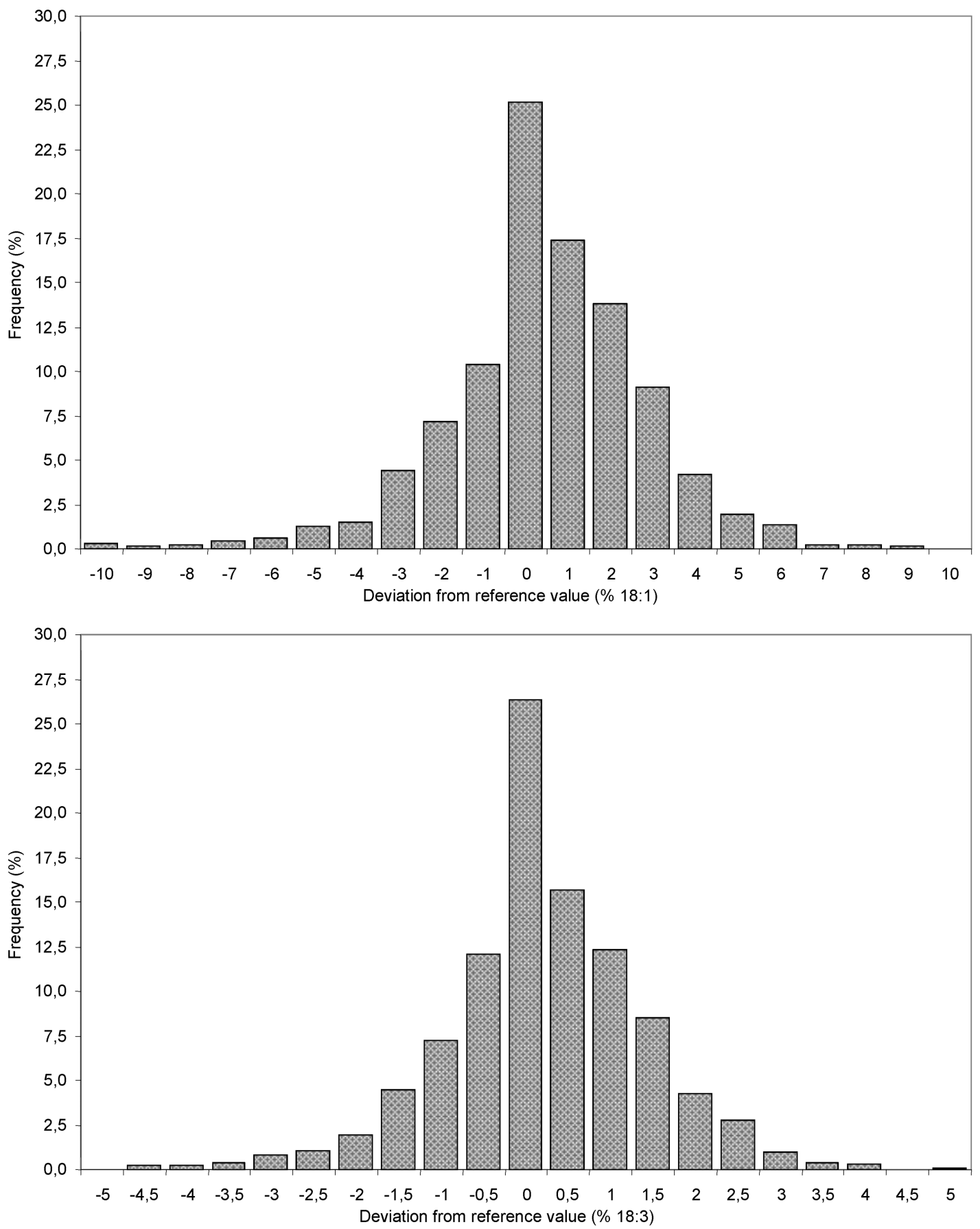

Figure 4. Frequency distribution of the absolute deviations of the predicted values for oleic acid and linolenic acid contents from their pertinent reference values determined by GC of single seeds of oilseed rape (calibration seed set with $n=3152$; LOCAL procedure).

approach, the correct fatty acid composition of the selected plant material has to be checked in subsequent generations by gas chromatography to meet the required quality standard.

For breeding purposes, it is furthermore relevant to know how well the fatty acid composition of F2 seeds correlates with the fatty acid composition of the derived F3 seeds. Applying gas chromatographic analysis, correlation coefficients between $0.71^{* *}$ (own unpublished results) and $0.76^{* *}$ have been found for oleic acid (7). For linolenic acid a correlation coefficient of $r=0.80^{* *}$ was reported (7).

In summary, the high-throughput single-seed NIRS method developed in the present work has shown to be useful for the selection of single-seed genotypes with a high oleic acid and low linolenic acid content. Further work has to show if the new single-seed NIRS method can be applied to screen for other seed quality traits.

\section{ACKNOWLEDGMENT}

Many thanks to Deutsche Saatveredelung AG (Lippstadt), KWS Saat AG (Einbeck), Norddeutsche Pflanzenzucht HansGeorg-Lembke KG (Hohenlieth), RAPS GbR Saatzucht Lundsgaard (Grundhof), and Syngenta Seeds GmbH (Bad Salzuflen) for providing seed material.

\section{LITERATURE CITED}

(1) Vollman, J.; Rajcan, I. Oil crop Breeding and Genetics. In Handbook of Plant Breeding 4; Vollman, J., Rajcan, I., Eds.; Springer Science and Business Media: Heidelberg, 2009; Chapter 1, pp 1-30.

(2) Barth, C. Nutritional value of rapeseed oil and its high oleic/low linolenic variety - A call for differentiation. Eur. J. Lipid Sci. Technol. 2009, 111, 953-956. 
(3) Möllers, C. Potential and future prospects for rapeseed oil. In Rapeseed and Canola oil-production, processing, properties and uses; Gunstone, F. D., Ed.; Blackwell Publishing: Oxford, UK, 2004; pp $186-217$.

(4) Scarth, R.; McVetty, P. B. M.; Rimmer, S. R.; Stefansson, B. R. 'Stellar', low linolenic-high linoleic acid summer rape. Can. J. Plant Sci. 1988, 68, 509-511.

(5) Scarth, R.; Rimmer, S. R.; McVetty, P. B. M. Apollo low linolenic summer rape. Can. J. Plant Sci. 1995, 75, 203-204.

(6) Lehmann, L.; Hamann, M.; Stelling, D.; Busch, H. Vistive ${ }^{\circledR}$ HOLLi-Winterraps. Raps 2008, 4, 208-211.

(7) Schierholt, A.; Becker, H. C. Entwicklung von Hochölsäure Raps. In Öl- und Faserpflanzen; Diepenbrock, W., Eissner, H., Eds.; UFOP-Schriften: Bonn, Germany, 2000; Vol. 14, pp 319-323.

(8) Schierholt, A.; Becker, H. C. Environmental variability and heritability of high oleic acid content in winter oilseed rape. Plant Breed. 2001, 120, 63-66.

(9) Rücker, B.; Röbbelen, G. Impact of low linolenic acid content on seed yield of winter oilseed rape (Brassica napus L.). Plant Breed. 1996, 115, 226-230.

(10) Velasco, L.; Becker, H. C. Estimating the fatty acid composition of the oil in intact-seed rapeseed (Brassica napus L.) by near-infrared reflectance spectroscopy. Euphytica 1998, 101, 221-230.

(11) Thies, W. Schnelle und einfache Analysen der Fettsäurezusammensetzung in einzelnen Raps-Kotyledonen. I. Gaschromatographische und papierchromatographische Methode. Z. Pflanzenzüchtg. 1971, $65,181-202$.

(12) Sato, T.; Uezono, I.; Morishita, T.; Tetsuka, T. Nondestructive estimation of fatty acid composition in seeds of Brassica napus L. by near-infrared spectroscopy. J. Am. Oil Chem. Soc. 1998, 75, 18771881.

(13) Velasco, L.; Möllers, C.; Becker, H. C. Estimation of seed weight, oil content and fatty acid composition in intact single seeds of rapeseed (Brassica napus L.) by near infrared reflectance spectroscopy. Euphytica 1999, 106, 79-85.

(14) Velasco, L.; Möllers, C. Nondestructive assessment of protein content in single seeds of rapeseed (Brassica napus L.) by nearinfrared reflectance spectroscopy. Euphytica 2002, 123, 89-93.
(15) Hom, N. H.; Becker, H. C.; Möllers, C. Non-destructive analysis of rapeseed quality by NIRS of small seed samples and single seeds. Euphytica 2007, 153, 27-34.

(16) Berzaghi, P.; Shenk, J. S.; Westerhaus, M. O. LOCAL prediction with near-infrared multi-product databases. J. Near Infrared Spectrosc. 2000, 8, 1-9.

(17) Dardenne, P.; Sinnaeve, G.; Baeten, V. Multivariate calibration and chemometrics for near infrared spectroscopy: which method? J. Near Infrared Spectrosc. 2000, 8, 229-237.

(18) Dowell, F. E.; Maghirang, E. B.; Baenziger, P. S. Automated SingleKernel Sorting to Select for Quality Traits in Wheat Breeding Lines. Cereal Chem. 2009, 86, 527-533.

(19) Spielbauer, G.; Armstrong, P.; Baier, J. W.; Allen, W. B.; Richardson, K.; Shen, B.; Settles, A. M. High-Throughput Near-Infrared Reflectance Spectroscopy for Predicting Quantitative and Qualitative Composition Phenotypes of Individual Maize Kernels. Cereal Chem. 2009, 86, 556-564.

(20) Tremolieres, A.; Dubaco, J. P.; Drapier, D. Unsaturated fatty acids in maturing seeds of sunflower and rape: regulation by temperature and light intensity. Phytochemistry 2001, 21, 41-45.

(21) Zhao, J.; Dimov, Z.; Becker, H. C.; Ecke, W.; Möllers, C. Mapping QTL controlling fatty acid composition in a doubled haploid rapeseed population segregating for oil content. Mol. Breed. 2008, 21, $115-125$.

(22) Fontaine, J.; Hörr, J.; Schirmer, B. Near-infrared reflectance spectroscopy enables the fast and accurate prediction of the essential amino acid contents in soy, rapeseed meal, sunflower meal, peas, fishmeal, meat meal products, and poultry meal. J. Agric. Food Chem. 2001, 49, 57-66.

Received for review August 12, 2009. Revised manuscript received November 17, 2009. Accepted November 19, 2009. This research project was funded by the Bundesministerium für Ernährung, Landwirtschaft und Verbraucherschutz (BMELV), through the Fachagentur Nachwachsende Rohstoffe e.V. and the Gemeinschaft zur Förderung der privaten deutschen Pflanzenzüchtung e.V. (GFP) (FKZ 22013804). 